\title{
A new scoring system for peritoneal metastasis in gastric cancer
}

\author{
Takashi Fujimura, Kaname Ishit, Katsunobu Oyama, Tomoharu Miyashita, Shinichi Kinami, Itasu Ninomiya, \\ Hirohisa Kitagawa, Sachio Fushida, Genichi Nishimura, Masato Kayahara, Koichi Shimizu, Tetsuo Ohta, \\ and KoICHI Miwa
}

Gastroenterologic Surgery, Division of Cancer Medicine, Graduate School of Medical Science, Kanazawa University, 13-1 Takaramachi, Kanazawa 920-8641, Japan

\begin{abstract}
Background. The degree of peritoneal metastasis (PM) of gastric cancer has been classified into four grades (P0, P1, P2, and P3) in the Japanese Classification of Gastric Carcinoma, First English Edition. However, the PM category in this version was so ambiguous in terms of volumetry that it was unlikely to estimate PM status correctly. We have developed a new volume scoring system for PM, modifying Sugarbaker's classification.

Methods. Eighty-five patients with gastric cancer with PM underwent surgery and/or laparoscopy at our hospital at Kanazawa University between January 1990 and December 2000. The grading (Gr) by the volume scoring system was decided according to the sum of scores for nine (in male) or ten (in female) abdominopelvic regions. These regions included left subphrenics, right subphrenics, left flank, right flank, pelvis, omenta, mesentery, small and large bowels, ovaries (only for females), and other intraabdominal organs. Volumetry for each region was carried out using the following categories: VSO, indicating the absence of cancer in a particular abdominopelvic region; VS1, indicating that tumor nodules are less than $10 \mathrm{~mm}$ in diameter; VS2, indicating tumors between 1 and $5 \mathrm{~cm}$ in diameter; and VS3, indicating that tumor is more than $5 \mathrm{~cm}$ in diameter or that an organ is coated by a mat of tumor (confluent disease). The scores for VSO, VS1, VS2, and VS3 were $0,1,2$, and 3 points, respectively. Total scores of 1 to 3 were the equivalent of $\mathrm{Gr}$ I, total scores of 4 to 6 were equivalent to Gr II, total scores of 7 to 9 were equivalent to Gr III, and total scores of more than 9 were equivalent to Gr IV.

Results. There was no significant difference between $\mathrm{P1}$ and P2, or between P1 and P3 in survival curves. But the $50 \%$ survival times of Gr I, Gr II, Gr III, and Gr IV were 1.23, 0.66, 0.67 , and 0.32 years, respectively. The prognosis of $G r I$ was significantly better than that of any other grades. Eight of the 28 patients $(29 \%)$ assessed as Gr I survived more than 2 years, whereas only 2 of the 57 (4\%) assessed as Gr II, Gr III, or Gr IV did so. Multivariate analysis by Cox's proportional hazard model revealed that the volume scoring, resection of primary
\end{abstract}

Offprint requests to: T. Fujimura

Received: February 26, 2003 / Accepted: May 12, 2003 tumor, and resection of peritoneal tumor were independent factors to predict prognosis.

Conclusion. These results showed that the volume scoring system was strongly related to the prognosis, and the patients assessed as Gr I had a great probability of cure by surgery.

Key words Peritoneal metastasis of gastric cancer - Volume scoring system · Sugarbaker's classification

\section{Introduction}

The most frequent cause of death in gastric cancer is peritoneal metastasis (PM), which is difficult to diagnose preoperatively (in spite of the development of diagnostic imaging) and difficult to cure completely with surgery or chemotherapy. Moreover, a therapeutic strategy has not yet been established, because there is no standardized assessment of PM suitable for statistical analysis. The degree of PM in gastric cancer was originally classified into four grades (P0, P1, P2, and P3) in the Japanese Classification of Gastric Carcinoma, First English Edition (the First Edition) [1], edited by Japanese Research Society for Gastric Cancer. This classification was changed to two categories, positive or negative PM, in the Japanese Classification of Gastric Carcinoma, Second English Edition (the Second Edition) [2], because there was no significant difference in prognosis between any two $\mathrm{PM}$ - positive groups $(\mathrm{P} 1, \mathrm{P} 2$, or P3) [3]. However, the PM category of the First Edition was so ambiguous in terms of volumetry that it was unlikely to lead to a correct estimate of PM status. For example, any major peritoneal metastasis to the peritoneum adjacent to the stomach was regarded as P1 metastasis, and only 2-mm nodules on the pelvic peritoneum were assessed as P2. The grading of PM in the Second Edition has been greatly simplified. According to this new edition, if a patient shows PM, however small the peritoneal tumor may be, the patient is catego- 
rized as stage IV, the final stage of this disease. Recently, cure-oriented surgery for stage IV gastric cancer has been abandoned by most surgeons. We fear, however, that surgeons may miss patients who have a chance of being cured or having their survival prolonged because of inadequate classification of PM in both editions: incompleteness in terms of volumetry in the First Edition and oversimplification in the Second Edition.

When confronted in the operating room with PM from gastric cancer, the clinician must make a decision regarding the possible risks and benefits of aggressive treatment versus best supportive care [4]. It is therefore essential that objective criteria are established by which any clinician can decide on the optimal treatment strategy. In the present study we developed a new semiquantitative scoring system for PM by modifying Sugarbaker's classification (Jacquet and Sugarbaker [5]), and showed that the patients assessed as Gr I with our volume scoring system had a high probability of being cured by surgery.

\section{Patients and methods}

A total of 735 patients with histologically proven gastric carcinoma underwent surgery and/or laparoscopy at the Department of Gastroenterologic Surgery of Kanazawa University between January 1990 and December 2000. Eighty-five $(11.6 \%)$ of the patients had peritoneal metastases; 50 were males and 35, females; aged from 14 to 84 years, with an average age of $61 \pm 14$ years. The clinical and pathological findings were based on the guidelines of the first edition of the Japanese Classification of Gastric Carcinoma (the First Edition), edited by the Japanese Research Society for Gastric Cancer. According to the First Edition, the degree of peritoneal metastasis is defined as: $\mathrm{P} 0$, no peritoneal metastasis; P1, metastases to the adjacent but not to the distant peritoneum; P2, a few metastases to the distant peritoneum; and $\mathrm{P} 3$, numerous metastases to the distant peritoneum. The adjacent peritoneum is defined as including the peritoneum of the lesser sac as well as the lesser and greater omenta.

Cytoreductive surgery for the primary tumor and the peritoneal tumor was performed in $66(78 \%)$ and 33 (39\%) patients, respectively (Table 1$)$. The resection of peritoneal tumor included picking-up resection of peritoneal nodules $(n=24)$ and subtotal peritonectomy $(n$ $=9$ ). Fifty-two patients (61\%) underwent intraperitoneal chemotherapy using cisplatin, mitomycin $\mathrm{C}$, and etoposide.

We assessed the distribution and the volume of the intraperitoneal tumors. Using a modified version Jacquet and Sugarbaker's classification [5], we divided

\section{Parietal peritoneum}

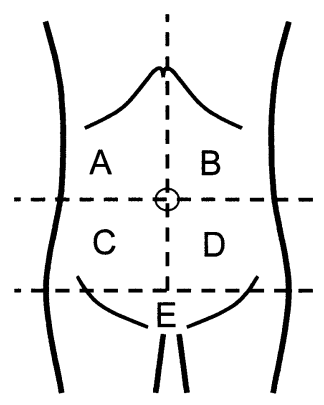

A Right subphrenics

B Left subphrenics

C Right flank

D Left flank

E Pelvis

Visceral peritoneum

F Major and minor omenta

G Mesentery

H Small and large bowels

I Ovary

$\mathrm{J}$ Other organs

Fig. 1. Abdominopelvic anatomic regions. The peritoneal surface was divided into ten abdominopelvic regions - five parietal and five visceral regions

the abdominopelvic anatomic sites into ten regions for the separate assessment of peritoneal tumors (Fig. 1). The abdominopelvic regions comprise five parietal and five visceral regions on the peritoneum. The parietal regions consist of the left subphrenics, right subphrenics, left flank, right flank, and pelvis. The upper transverse plane is the lowest aspect of the costal margin and the lower transverse plane is the aspect of the anterior superior iliac spine. The visceral regions consist of the minor and major omenta, mesentery, small and large bowels, ovaries (only for females), and other intraabdominal organs.

For volumetric scoring for each region, we used the following categories: VSO, absence of cancer in a particular abdominopelvic region; VS1, tumor nodules less than $10 \mathrm{~mm}$ in diameter; VS2, tumors between 1 and $5 \mathrm{~cm}$ in diameter; and VS3, tumors more than $5 \mathrm{~cm}$ in diameter or an organ coated by a mat of tumors (confluent disease) (Fig. 2A,B). The scores for VS0, VS1, VS2, and VS3 were $0,1,2$, and 3 points, respectively. Grading (Gr) based on volumetric scoring was decided according to the sum of the scores for nine (in males) or ten (in females) abdominopelvic regions. A total score of 1 to 3 was the equivalent of Gr I, a total score of 4 to 6 was equivalent Gr II, a total score of 7 to 9 was equivalent to Gr III, and a total score of more than 9 was equivalent to Gr IV.

Significant differences in proportions were determined with the $\chi^{2}$ test. Patient survival was calculated by the Kaplan-Meier method, and survival curves were compared by the log rank method. Multivariate analysis for prognostic values was carried out using Cox's proportional hazards regression model. Statistical significance was defined as a $P$ value of less than 0.05 . 
Table 1. Patient characteristics

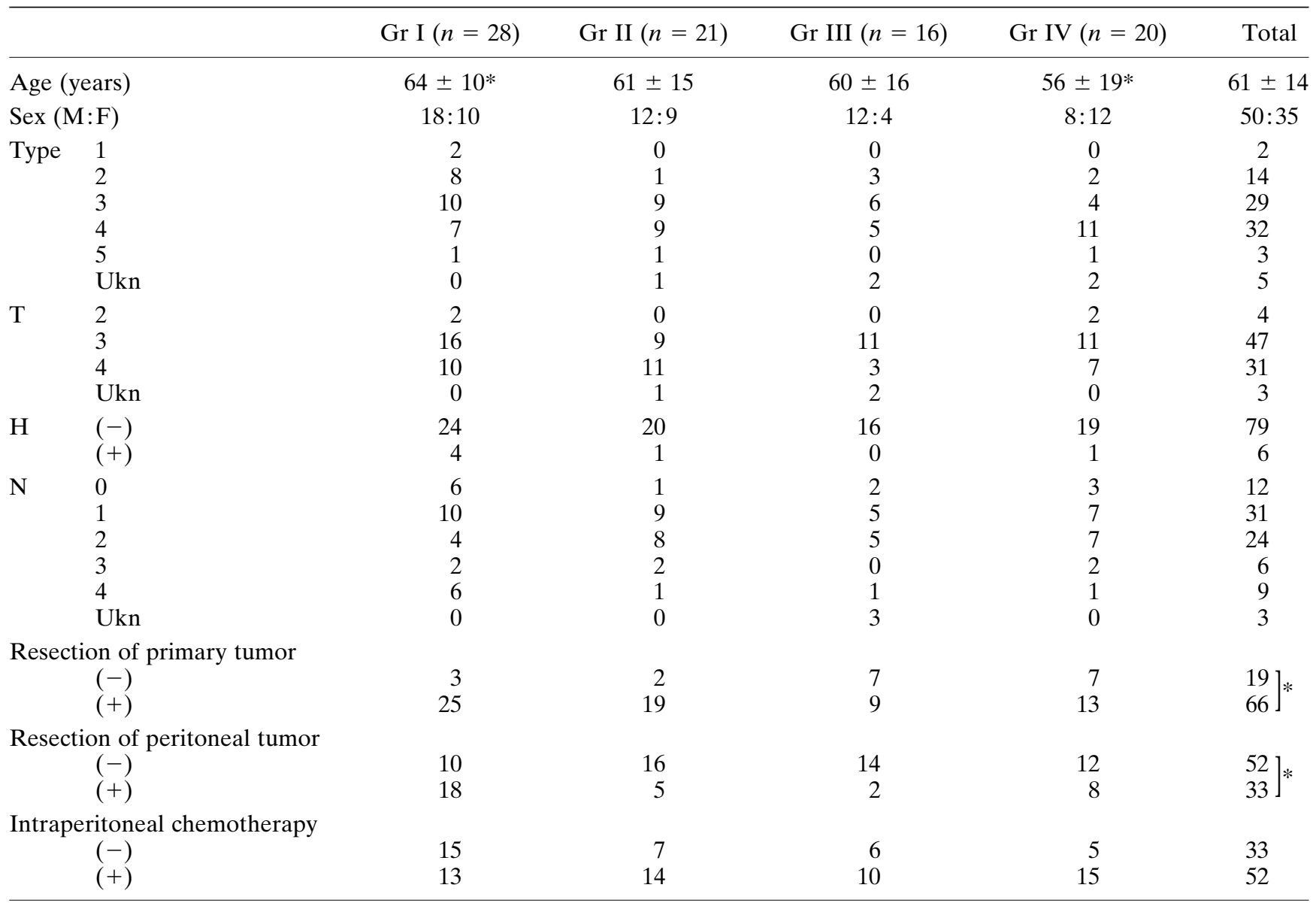

$* P<0.05$

$\mathrm{T}$, depth of tumor invasion; $\mathrm{H}$, liver metastases; $\mathrm{N}$, lymph node metastases; Ukn, unknown

\section{Results}

Our assessment resulted in categorizing 28 patients with Gr I, 21 with Gr II, 16 with Gr III, and 20 with Gr IV. The patients with Gr IV were significantly younger than those with Gr I (Table 1). The lower the grade of PM was, the more often resections of the primary tumor and the peritoneal tumor were performed. No statistically significant relationship could be established between status of the macroscopic type, depth of tumor invasion ( $\mathrm{T}$ factor), liver metastases ( $\mathrm{H}$ factor), lymph node metastases ( $\mathrm{N}$ factor), or intraperitoneal chemotherapy. The classification by the First Edition (P1, P2, and P3) was compared with the grading by the volume scoring system. All the patients assessed as P1 were categorized as Gr I. Sixteen of the 30 patients assessed as P2 were categorized as Gr I, 11 as Gr II, 2 as Gr III, and 1 as Gr IV. The patients assessed as $\mathrm{P} 3$ comprised 1 in Gr I, 10 in Gr II, 14 in Gr III, and 19 in Gr IV. The volumetric scoring (degree) and distribution of PM are shown in Table 2. Peritoneal tumor was found on mesentery $(64 \%)$, pelvis $(55 \%)$, left subphrenics $(39 \%)$, right subphrenics (35\%), omenta (35\%), right flank (34\%), left flank $(33 \%)$, bowels $(25 \%)$, ovaries $(23 \%)$, and other organs $(13 \%)$ in order of frequency. VS2 (tumors between 1 and $5 \mathrm{~cm}$ in diameter) appeared the most frequent of VS categories at all of the abdominopelvic anatomic sites.

The $50 \%$ survival times for the $\mathrm{P} 1, \mathrm{P} 2$, and $\mathrm{P} 3$ categories according to the First Edition were 1.14, 0.92, and 0.32 years, respectively (Fig. 3). There was no significant difference in survival between $\mathrm{P} 1$ and $\mathrm{P} 2$, or between $\mathrm{P} 1$ and $\mathrm{P} 3$, while the survival of the $\mathrm{P} 2$ patients was significantly better than that of the $\mathrm{P} 3$ patients $(P<0.001)$. Survival analysis according to the volumetric scoring system showed that $50 \%$ survival times for Gr I, Gr II, Gr III, and Gr IV were 1.23, 0.66, 0.67, and 0.32 years, respectively. The prognosis in Gr I was significantly better than that in the other grades, including Gr II, III, and IV (Fig. 4). Eight of the 28 patients (29\%) assessed as Gr I survived for more than 2 years, whereas only 2 of the 57 (4\%) assessed as Gr II, Gr III, or Gr IV did so. Next, we examined whether resection of the primary tumor was effective for prolonging the survival of Gr I 
A

\begin{tabular}{|c|c|c|c|c|}
\hline Degree & VSO & VS1 & VS2 & VS3 \\
\hline \multicolumn{5}{|c|}{ Tumor size } \\
\hline & No & $\sim 1 \mathrm{~cm}$ & $1 \sim 5 \mathrm{~cm}$ & $>5 \mathrm{~cm}$ or confluent \\
\hline Point & 0 & 1 & 2 & 3 \\
\hline
\end{tabular}

B

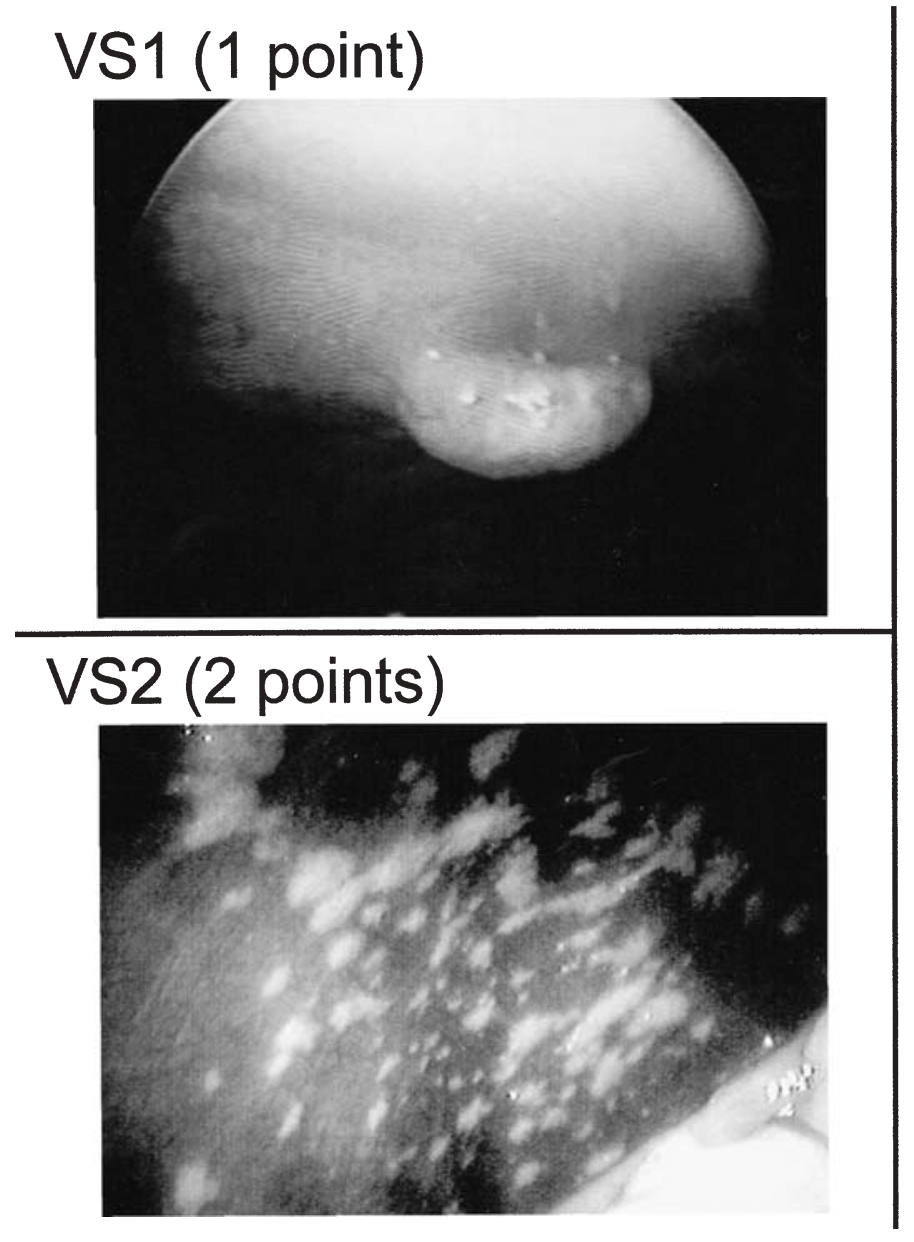

VS3 (3 points)
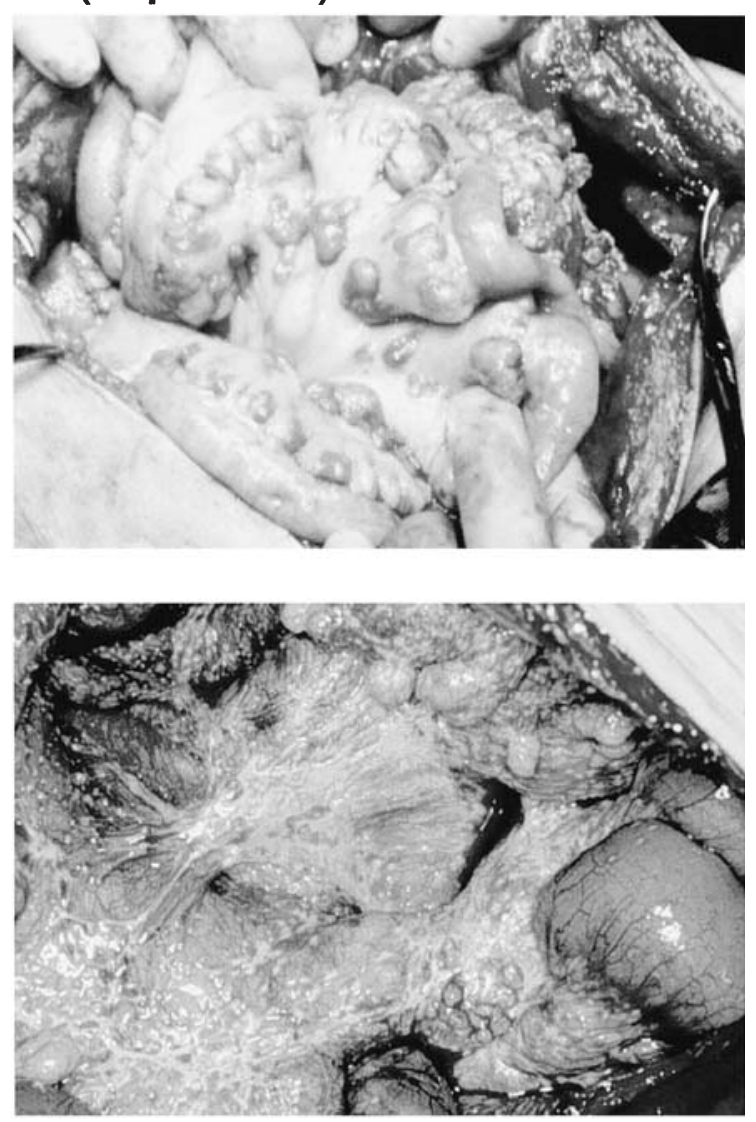

Fig. 2. A Volume scoring system for peritoneal metastasis (modification of classification by Jacquet and Sugarbaker [5]). The degree for the volume scoring was semiquantitatively assessed according to the size of the peritoneal metastasis. Degrees of VS0, VS1, VS2, and VS3 were scored as $0,1,2$, and 3 points, respectively. Points for volume scoring were added for all regions (regions shown in Fig. 1). B Representative photographs of peritoneal metastases according to volume scoring. The volume scoring was performed during laparotomy or laparoscopy

patients (Fig. 5). As expected, the survival of the patients assessed as Gr I who had undergone resection of the primary tumor was much better than that of patients who had not $(P=0.01)$.

Multivariate analysis by Cox's proportional hazards model revealed that the volume scoring $(\mathrm{Gr}$ II + III +
IV/Gr I) (odds ratio, 3.18; 95\% confidence interval [CI], $1.56-6.49 ; P=0.0015)$; resection of primary tumor (odds ratio, 0.44; 95\% CI, 0.22-0.90; $P=0.023$ ); and resection of peritoneal tumor (odds ratio, $0.50 ; 95 \% \mathrm{CI}$, $0.25-0.98 ; P=0.043$ ) were independent prognostic factors (Table 3). However, the PM classification of the 
Table 2. The frequency and distribution of peritoneal metastasis

\begin{tabular}{|c|c|c|c|c|c|}
\hline \multirow[b]{2}{*}{ Site } & \multicolumn{4}{|c|}{ Volumetric scoring } & \multirow[b]{2}{*}{ Frequency (\%) } \\
\hline & VS0 & VS1 & VS2 & VS3 & \\
\hline Right subphrenics & 55 & 3 & 27 & 0 & $30(35)$ \\
\hline Left subphrenics & 52 & 5 & 26 & 2 & $33(39)$ \\
\hline Right flank & 56 & 8 & 20 & 1 & $29(34)$ \\
\hline Left flank & 57 & 9 & 19 & 0 & $28(33)$ \\
\hline Pelvis & 38 & 11 & 32 & 4 & $47(55)$ \\
\hline Omenta & 55 & 6 & 18 & 0 & $30(35)$ \\
\hline Mesentery & 31 & 14 & 35 & 6 & $54(64)$ \\
\hline Bowels & 64 & 1 & 15 & 5 & $21(25)$ \\
\hline Ovarya & 27 & 0 & 6 & 2 & $8(23)$ \\
\hline Other organs & 74 & 4 & 7 & 0 & $11(13)$ \\
\hline
\end{tabular}

${ }^{a}$ Only for females

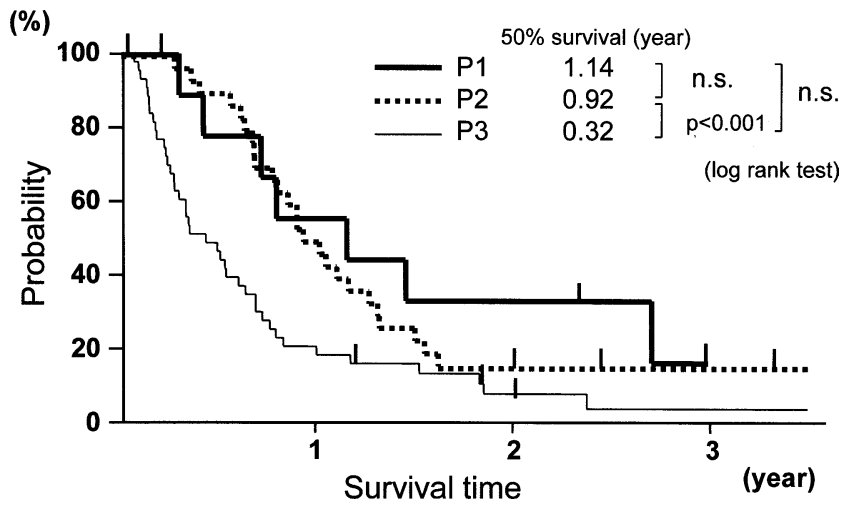

Fig. 3. Survival curves determined according to the classification in the first English edition of the Japanese classification of gastric carcinoma. The $50 \%$ survival times of the P1, P2, and P3 categories were $1.14,0.92$, and 0.32 years, respectively. There was no significant difference (n.s.) in survival between $\mathrm{P} 1$ and $\mathrm{P} 2$, or between $\mathrm{P} 1$ and $\mathrm{P} 3$

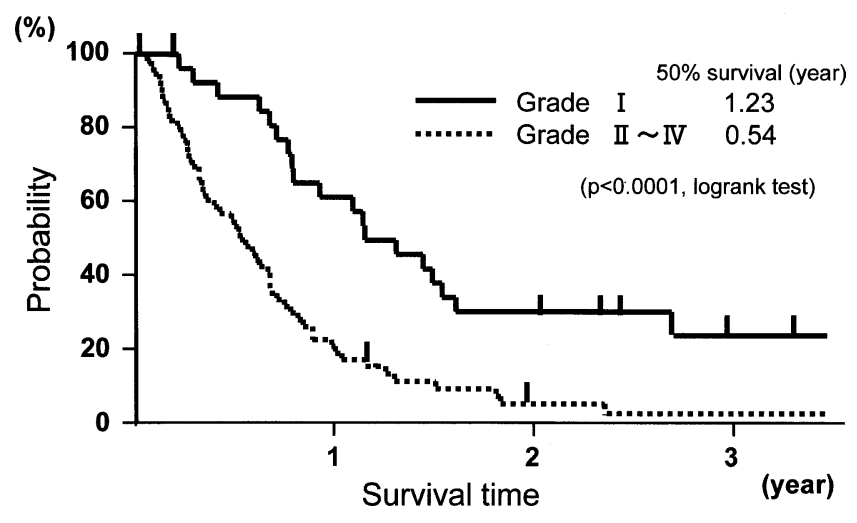

Fig. 4. Survival curves determined by the grading in the volume scoring system. The $50 \%$ survival time of Gr I was 1.23 years, while that of the other grades, including Gr II, III, and IV, was 0.54 years. The prognosis of Gr I was significantly better than that of the other grades

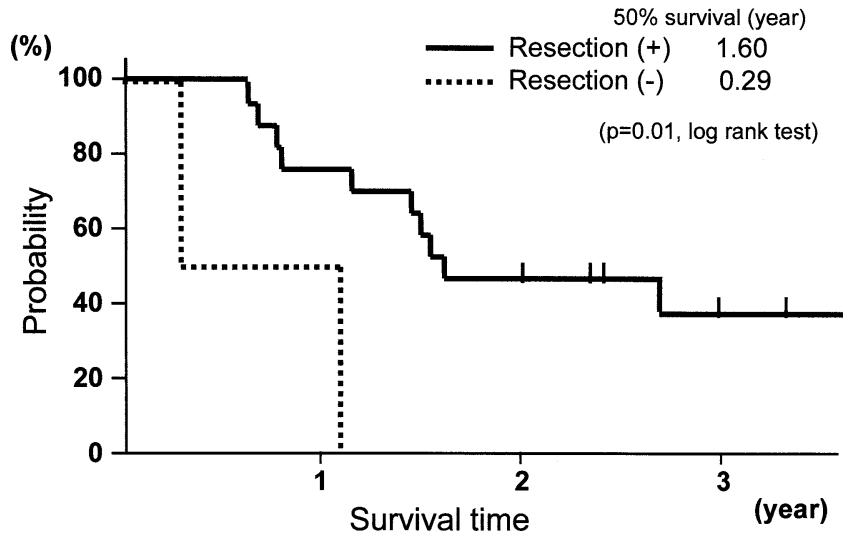

Fig. 5. Survival curves of patients assessed as Gr I, according to resection of the primary tumor. Patients with metastases to liver and/or paraaortic lymph nodes were excluded from the analysis. The 1-year and 2-year survival rates of the patients who underwent resection were $76 \%$ and $47 \%$, significantly better than the rates of $50 \%$ and $0 \%$ for the patients who did not undergo resection (log rank test, $P=0.01$ )

First Edition (P2 + 3 / P1) (odds ratio, 0.51; 95\% CI, $0.18-1.44 ; P=0.20$ ) was not found to be a predictor of survival. Paraaortic lymph node metastasis and cytology showed marginal importance as prognostic factors.

\section{Discussion}

For most malignant tumors the prognosis depends upon the size or volume of the primary tumor. It is only natural to choose these as one of the prognostic factors for survival analysis. The PM categories in the First Edition of the Japanese classification of gastric carcinoma are so ambiguous in terms of volumetry, however, that it is unlikely that the status of PM can be correctly estimated. The four degrees of PM (P0, P1, P2, and P3) 
Table 3. Multivariate analysis for prognostic factors

\begin{tabular}{|c|c|c|c|}
\hline Factor & Odds ratio & $\begin{array}{l}\text { \% Confidence } \\
\text { interval }\end{array}$ & $P$ value \\
\hline Age & 1.00 & $0.98-1.02$ & 0.66 \\
\hline Liver metastasis $(+/-)$ & 1.49 & $0.67-3.34$ & 0.33 \\
\hline Paraaortic lymph node metastasis $(+/-)$ & 2.03 & $0.88-4.72$ & 0.10 \\
\hline $\mathrm{P}(\mathrm{P} 2 \text { plus } \mathrm{P} 3 / \mathrm{P} 1)^{\mathrm{a}}$ & 0.51 & $0.18-1.44$ & 0.20 \\
\hline $\begin{array}{l}\text { Volume scoring } \\
\text { (grades II, III, and IV / grade I) }\end{array}$ & 3.18 & $1.56-6.49$ & 0.0015 \\
\hline Cytology $(+/-)$ & 1.53 & $0.86-2.72$ & 0.14 \\
\hline Resection of primary tumor $(+/-)$ & 0.44 & $0.22-0.90$ & 0.023 \\
\hline Resection of peritoneal tumor $(+/-)$ & 0.50 & $0.25-0.98$ & 0.043 \\
\hline Intraperitoneal chemotherapy $(+/-)$ & 1.04 & $0.55-1.96$ & 0.91 \\
\hline
\end{tabular}

defined in this edition were not determined by measuring the peritoneal tumor burden or by any other objective methods. When the Japanese classification of gastric carcinoma was revised in 1998 to accord with the TNM classification of The International Union Against Cancer (UICC) [6], this classification for PM was abandoned because there was no significant difference in prognosis between any two PM-positive groups (P1, P2, or P3) [3]. The degree of peritoneal metastasis in the Second Edition is simply defined as: P0, no peritoneal metastasis; P1, peritoneal metastasis; and PX, unknown. The M-category in the TNM classification includes all types of distant metastases, such as those in liver, lung, and peritoneum, while in the Second Edition, the "P" and " $\mathrm{H}$ " categories are independent of the " $\mathrm{M}$ " category. However, the categorization of PM in the Second Edition has become very similar to that of the "M" category of the TNM classification. The problem is that assessment of the tumor burden of the PM is completely omitted from both classifications; that is, the presence or absence of PM is the only determinant, regardless of the size, volume, and distribution of PM. It seems that no quantitative estimation of PM has been reported, except for Jacquet and Sugarbaker's classification [5]. We previously developed and used a classification that grouped the macroscopic features of PM into three types; tubercular, nodal, and diffuse [7]. However, there was no significant difference in survival between these three types. We, therefore, planned this study to show the importance of volumetry of PM in gastric cancer.

The rationale for palliative operation for patients with PM is still controversial. Ouchi et al. [8] suggested that gastrectomy should be performed for patients with a minimal amount of PM (P1), and that resection for moderate or extensive PM (P2 or P3) had no beneficial effect on survival or quality of life. On the other hand, another author has stated that the extent of PM did not affect prognosis and that palliative gastrectomy was indicated for patients, regardless of the extent of PM, if the primary tumor was surgically resectable and without any evidence of liver metastases [9]. Furthermore, subtotal peritonectomy has been recommended for patients with PM at a few institutes [10]. But the indications for resection and the operative procedures in the surgical strategy for the treatment of PM remain to be clarified. In the present study, we provided evidence that, if the volume of the peritoneal tumors was as small as Gr I, the patient had a high probability of good prognosis and cure-oriented surgery should be carried out. It is likely that our classification will help to confirm the application and the benefits of resection of primary tumors for patients with PM.

Our classification should be used for macroscopic and histological findings, and not for radiological or ultrasonographic methods. Jacquet et al. [11] demonstrated that diagnostic imaging showed only 50\% accuracy for lesions from 0.5 to $5 \mathrm{~cm}$ in diameter, while above $5 \mathrm{~cm}$ the accuracy was $90 \%$. Exploratory laparoscopy is a less invasive method to assess PM than open laparotomy, and laparoscopy matches laparotomy in capacity to diagnoste PM [12]. Our diagnostic and therapeutic strategies for PM are as follows. When preoperative laparoscopy discloses a small amount of PM such as Gr I, cure-oriented surgery, including resection of the primary tumor and partial peritonectomy (of the region bearing peritoneal tumors) is performed. But when massive PM is present, anticancer agents, such as various combinations of 5-fluorouracil, mitomycin $\mathrm{C}$, cisplatin, and etoposide are administered systemically and intraperitoneally as neoadjuvant chemotherapy. If the chemotherapy is effective, laparoscopy is carried out again. If second-look laparoscopy demonstrates complete or near complete remission of PM, cytoreductive surgery is considered; if not, second-line chemotherapy is tried, using docetaxel or paclitaxel. 


\section{References}

1. Japanese Research Society for Gastric Cancer. Japanese classification of gastric carcinoma. First English Ed. Tokyo: Kanehara; 1995.

2. Japanese Research Society for Gastric Cancer. Japanese classification of gastric carcinoma. Second English Ed. Tokyo: Kanehara; 1998.

3. Aiko T, Sasako M, General Rules Committee of the Japanese Gastric Cancer Association. The new Japanese classification of gastric carcinoma: points to be revised. Gastric Cancer 1998;1:2530 .

4. Sugarbaker PH, Yonemura Y. Clinical pathway for the management of resectable gastric cancer with peritoneal seeding: best palliation with a ray of hope for cure. Oncology 2000;58:96-107.

5. Jacquet P, Sugarbaker PH. Clinical research methodologies in diagnosis and staging of patients with peritoneal carcinomatosis. In: Sugarbaker $\mathrm{PH}$, editor. Peritoneal carcinomatosis: principles of management. Boston: Kluwer Academic; 1996. pp. 359-74.

6. International Union Against Cancer. Sobin LH, Wittekind Ch TNM classification of malignant tumors. 5th Ed. Heidelberg Berlin Tokyo New York: Springer-Verlag; 1997.
7. Fujimura T, Yonemura Y, Kawamura T, Nojima N, Satoh T, Hirono Y, et al. Cytoreductive surgery and sandwich therapy with chemohyperthermic peritoneal perfusion and intra-aortic chemotherapy for peritoneal dissemination in gastric cancer. Oncol Rep 1996:3:513-7.

8. Ouchi K, Sugawara T, Ono H, Fujiya T, Kamiyama Y, Kakugawa $\mathrm{Y}$, et al. Therapeutic significance of palliative operations for gastric cancer for survival and quality of life. J Surg Oncol 1998; 69:41-4.

9. Kikuchi S, Arai S, Morise M, Kobayashi N, Tsukamoto H, Shimao H, et al. Gastric cancer with metastases to the distant peritoneum: a 20-year surgical experience. Hepatogastroenterology 1998;45:1183-88.

10. Sugarbaker PH. Peritonectomy procedure. Ann Surg 1995;221: 29-42.

11. Jacquet P, Jelinek JS, Steves MA, Sugarbaker PH. Evaluation of computed tomography in patients with peritoneal carcinomatosis. Cancer 1993;72:1631-6.

12. Fujimura T, Kinami S, Ninomiya I, Kitagawa H, Fushida S, Nishimura G, et al. Diagnostic laparoscopy, serum CA125, and peritoneal metastasis in gastric cancer. Endoscopy 2002;34:569-74. 